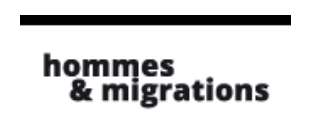

Hommes \& migrations

Revue française de référence sur les dynamiques

migratoires

$1297 \mid 2012$

Migrations en création

\title{
L'immigration dans le cinéma français et québécois à travers quelques films
}

\section{Concepción López-Campos Bodineau}

\section{(apenEdition \\ Journals}

\section{Édition électronique}

URL : http://journals.openedition.org/hommesmigrations/1541

DOI : 10.4000/hommesmigrations. 1541

ISSN : 2262-3353

\section{Éditeur}

Musée national de l'histoire de l'immigration

\section{Édition imprimée}

Date de publication : 1 mai 2012

Pagination : 20-28

ISSN : 1142-852X

\section{Référence électronique}

Concepción López-Campos Bodineau, «L'immigration dans le cinéma français et québécois à travers quelques films », Hommes \& migrations [En ligne], 1297 | 2012, mis en ligne le 31 décembre 2014, consulté le 19 avril 2019. URL : http://journals.openedition.org/hommesmigrations/1541 ; DOI : 10.4000/hommesmigrations.1541 


\section{L'immigration dans le cinéma français et québécois à travers quelques films}

Par Concepción López-Campos Bodineau, chargée de cours à l'université Aix-Marseille

Dans son traitement cinématographique, l'image de l'immigré est chargée d'un pouvoir critique, celui des politiques migratoires qui encadrent sa présence sur le sol de son pays d'accueil. En France, par la voix de l'immigré ou de ses enfants se disent les difficultés de

la double appartenance, de l'adaptation à la culture majoritaire. Au Québec, qui a choisi une politique multiculturaliste, se font jour les tentations au repli communautaire. Des thématiques différentes qui témoignent des difficultés récurrentes de l'intégration. 
Nous voulons présenter quelques films qui, selon nous, offrent une image intéressante des problèmes liés à l'immigration en France et au Québec. Cette image est le reflet d'une partie de la société, celle des émigrés, représentée de manière subjective à travers la vision qu'en ont les différents metteurs en scène.

En effet, selon Michèle Lagny: "Le cinéma est, au même titre que la peinture ou la sculpture, un mode d'expression du groupe dans lequel il est créé et à travers lequel se transmettent de nombreuses idées et valeurs ancrées consciemment ou inconsciemment dans notre sociétét ${ }^{(1)}$."

Au cours de ces dernières années, on a assisté à l'apparition de multiples films prenant pour thème l'immigration et mettant en scène des immigrés de la première ou de la deuxième génération. Certains de ces films donnent une vision quelque peu idyllique de l'immigré que l'on voit s'adapter facilement à son pays d'accueil, qui trouve facilement où se loger et ne souffre pour ainsi dire pas du racisme. C'est le cas du film d'Henri Verneuil, Mayrig (1991). On trouve également des films qui offrent une vision plus légère de la situation des immigrés et de leurs enfants, comme le film français Beur, Blanc, Rouge de Mahmoud Zemmouri (2005) ou Comment conquérir l'Amérique, le film québécois de Dany Lafarrière (2004). Cependant, la plupart des films dépeignent une réalité beaucoup plus dure et difficile pour l'immigré, confronté à la précarité du travail, aux problèmes de logement, à la propre difficulté d'adaptation à la société d'accueil et parfois même au racisme qui s'exerce à son encontre.

Les enfants des travailleurs immigrés, ceux que l'on appelle "de la deuxième génération", occupent une place importante. C'est à travers eux que l'on découvre l'histoire de leurs pères et ce sont eux qui souffrent le plus des conséquences de l'inadaptation de la famille à son nouvel environnement. Si les parents sont marqués par la rupture qu'impose l'exil, les enfants vont devoir ancrer leurs racines dans la société qui les a vu naître et affronter le douloureux problème de l'intégration/assimilation qu'ils résoudront plus ou moins facilement selon le contexte historique du moment ${ }^{(2)}$.

Nous essaierons de montrer les différentes visions des immigrés qui nous sont proposées, à travers le regard qu'ont porté plusieurs metteurs en scène sur les problèmes posés par l'immigration, en France et au Québec. Nous nous référerons à quelques films qui nous ont paru représentatifs, en retenant deux axes principaux : les jeunes d'origine étrangère et les femmes migrantes.

\section{Les jeunes d'origine étrangère}

Beur, Blanc, Rouge (2005), le film de Mahmoud Zemmouri, évoque, sur le mode humoristique, les problèmes d'intégration que connaissent les enfants des immigrés nés en France, ceux que l'on appelle "de la deuxième génération", qui ont la plus grande difficulté à se situer entre le pays d'origine de leurs parents et celui qui a 
accueilli ces derniers mais qui les a vu naître. Le film souligne, chez ces jeunes, ce déchirement dû à leur double appartenance qui apparait lors d'un match amical de football qui a opposé l'Équipe de France qui venait d'être sacrée championne du monde et que l'on avait précisément appelée Beur, Blanc, Rouge du fait de ses nombreux joueurs "issus de la diversité" à celle d'Algérie que le jeune public avait majoritairement soutenue, envahissant même le terrain en brandissant des drapeaux algériens. Le jeune "beur" protagoniste de cette histoire, qu'on avait vu brandir le drapeau algérien lors de ce match, voudra plus tard accompagner ses parents en Algérie mais, au moment de franchir la frontière, il se verra refouler par les autorités algériennes car, né en France et donc de nationalité française, il n’a pas de visa. Le sentiment d'être entre deux histoires, deux cultures, deux pays et de n'appartenir entièrement à aucun s'impose à lui. Il lui restera comme seul refuge le quartier où il est né, sa cité et ses amis.

On retrouve ce même problème d'appartenance dans le film de Christophe Ruggia, Le Gone du Chaâba (1997). Cette fois, il s'agit des difficultés que rencontre le protagoniste, né en France, dans sa relation avec son frère aîné qui est né en Algérie et même avec ses camarades de collège qui le repoussent parce qu'il a de bons résultats et l'accusent à cause de cela d'être "français". Tout ceci amène ces jeunes garçons à se poser des questions sur leur identité, suis-je français ? algérien ? arabe ? Cela entrầnera pour eux une autre difficulté, celle d'accepter les institutions du pays d'accueil et de s'y adapter. Peu nombreux, comme nous le montre le film, sont ceux qui se rendront compte que, pour sortir de la misère et avoir une vie moins dure, ils devront étudier et, en quelque sorte, accepter l'assimilation dans le système français.

Pourtant, les familles font leur possible pour maintenir bien vivants les souvenirs et les coutumes du pays lointain d'où ils viennent et dans lequel la plupart d'entre eux ne retourneront pas, au prix même parfois de quelque transgression des lois. C'est ainsi qu'on voit l'installation d'une boucherie clandestine où l'on sacrifiera les animaux selon le rite halal, et qui sera démantelée par la police, ou encore la célébration du rituel de la circoncision des jeunes garçons.

Ce problème de double appartenance n'apparaît pas de la même façon dans les films québécois.

Dans la série de films qui pose le problème de l'immigration, il nous a paru intéressant de montrer la vision qu'en donne le film québécois Comment conquérir l'Amérique, de Dany Laferrière, vision quelque peu amère mais divertissante des immigrés au Québec. Le film nous montre l'arrivée au Québec d'un jeune haïtien, venu avec l'intention de s'y installer. Ce jeune homme est émerveillé par la société de consommation qu'il découvre et ébloui par son opulence. On retiendra la séquence où le protagoniste se met à jeter la nourriture en disant qu'ici on peut 
gaspiller les aliments et même les jeter si l'on veut, sans se préoccuper de savoir si le lendemain on aura de quoi manger ou non. En revanche, son oncle, qu'il a rejoint, nous montre la face amère du déracinement. Il est déçu par le pays et sa maison est envahie de cartons, comme s'il n'avait pas fini de déménager. Toutes ses pensées sont tournées vers un possible et prochain retour à Haïti. En attendant cet éventuel départ, comme dans Le Gône de Châa$b a$, l'oncle s'efforce de maintenir les traditions de son pays d'origine dans sa façon de manger ou de s'habiller. Ces deux personnages reflètent deux attitudes bien différentes

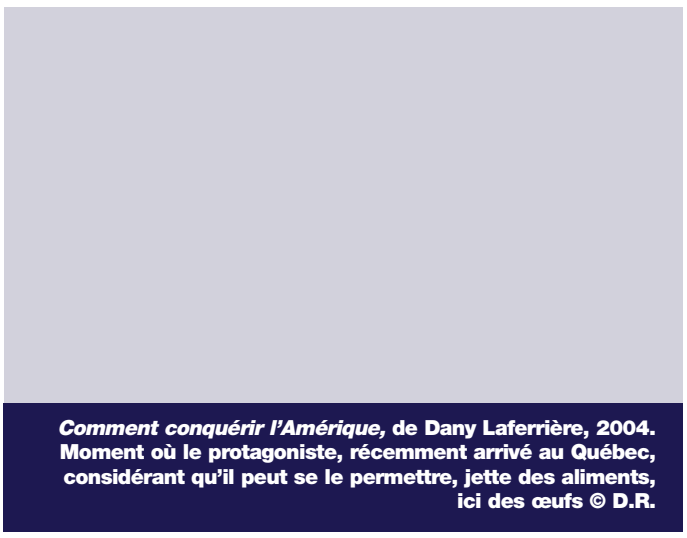
des immigrants, l'oncle n'a pas été capable de s'adapter et rejette en bloc le pays d'accueil, tandis que le neveu rêve de s'adapter, de s'intégrer, de s'assimiler et d'oublier son pays d'origine. Nous retrouverons plus loin, dans d'autres films québécois, cette même attitude qui est rarement transposée dans les films français.

C'est que, dans les films québécois, le problème d'adaptation ne se pose pas aussi souvent que dans les films français, car la législation sur l'immigration est bien différente. En France la politique officielle vis-à-vis des immigrés les pousse à s'assimiler et à s'intégrer. Au Québec, la politique menée par le gouvernement central du Canada est le multiculturalisme qui a eu comme effet l'apparition du communautarisme, de sorte que, par exemple, la ville de Montréal est tacitement divisée en quartiers ethniquement distincts (italien, algérien, espagnol...).

Cette division est bien visible dans le film québécois Mambo Italiano, d'Émile Gaudreault (2004), où les situations induites par le communautarisme sont bien représentées. Ainsi, on voit un fils d'immigré italien dont le problème ne vient pas d'une difficulté à s'adapter au pays d'accueil, puisqu'il habite dans le quartier italien où tout le monde est italien et parle italien, mais au contraire de rompre avec la tradition italienne et de quitter son quartier pour pouvoir s'intégrer pleinement dans le pays d'accueil. Ce film nous donne un exemple, peut-être un peu exagéré, du communautarisme au Québec et des problèmes qu'il provoque dans la société.

Cette opposition entre la vie d'un enfant d'immigré immergé dans son quartier, dans sa communauté, et sa vie à l'extérieur où il peut être totalement intégré à la vie québécoise se retrouve dans le film de Denis Chouinard, L'Ange du Goudron (2001). 


\section{La place des femmes}

$\mathrm{Si}$, comme nous venons de le voir, les films portant sur les problèmes des immigrés font une large place aux enfants et à leurs difficultés à s'insérer dans le pays d'accueil de leurs parents, un autre personnage occupe également une place importante dans ces mêmes films, français ou québécois, c'est le personnage de la femme, sa place et son rôle dans le processus d'intégration, comme épouse, comme mère ou tout simplement comme femme.

Dans la plupart des films français, de façon directe ou indirecte, on voit la présence importante de la femme pour maintenir l'ordre et la tradition. Nous choisirons comme exemples les films de Christophe Ruggia, Le Gone du Chaâba (1997), de Bourlem Guerdjou, Vivre au paradis (1997), et de Yamina Benguigui, Inch'Allah dimanche (2007).

Dans le premier, le sujet est traité à partir de la perspective d'un enfant, le dernier fils d'une famille d'immigrés algériens, et le seul né en France, qui vivent dans un "bidonville" à Lyon. Le second film nous fait voir la

Le Gone du Chaâba de Christophe Ruggia (1997). Le protagoniste et un ami revêtus des habits traditionnels pour la circoncision qui marque le passage de l'enfance
à l'âge adulte dans la tradition familiale $\odot$ D.R. vie d'adultes, mais cette fois dans les "bidonvilles" de la périphérie parisienne. Le troisième nous amène à Saint-Quentin, en Picardie, où on voit les difficiles démarches pour parvenir à la réunification familiale. Comme exemples québécois, on évoquera les films De ma fenêtre sans maison (Maryanne Zéhil, 2006) et Serveuses demandées (Guylaine Dionne, 2009) qui nous montrent des aspects très intéressants de l'immigration des femmes. $\mathrm{Si}$, dans Le Gone du Chaâba les protagonistes principaux sont les enfants, les personnages de femmes sont également intéressants. Les femmes ne sortent pas du bidonville, elles ne parlent que l'arabe, se chargent de toutes les tâches ménagères et organisent toutes les cérémonies coutumières, comme la circoncision des jeunes garçons. Les femmes comme on le voit ici ne sont absolument pas intégrées dans le pays d'accueil et ne cherchent pas à l'être.

En 1997, le film de Bourdem Guerdjou, Vivre au paradis, inspiré de la dureté de la vie dans les bidonvilles des années soixante, nous offre une vision plus âpre de la situation souvent dramatique des immigrés. Ici, il s'agit d'un travailleur algérien qui, dans le 
cadre du regroupement familial, fait venir sa famille. Alors que la femme arrive dans un pays censé être plus libéral et moderne que le sien, elle doit subir pourtant l'autorité du mari qui veut la confiner dans une situation d'isolement. Interdiction de sortir de la maison, d'aller faire les courses, d'établir des relations avec d'autres femmes du bidonville, et obligation de réaliser à l'intérieur de la "maison" tous les travaux ménagers.

Cependant cette femme peu à peu arrive à s'affranchir de la domination de son mari et, si elle est dans ce film aussi la gardienne des traditions du pays dans la vie familiale, elle participe à la vie du bidonville et s'implique même dans la lutte que mène le FLN. Peut-être faut-il voir à travers son personnage l'évolution plus générale des femmes immigrées.

On retrouve des problèmes similaires dans le film de Yamina Benguigui, Inch'Allah dimanche (2007). La situation de la femme est identique, elle arrive dans un pays étranger et son mari la confine chez elle. À la différence de Vivre au paradis, elle vit avec son mari, ses deux enfants et sa belle-mère dans un appartement avec un petit jardin que le mari a pu obtenir et dont les voisins ne sont pas des immigrés mais des Français. Au début, elle n'a pas le droit de sortir et elle doit même endurer les mauvais traitements physiques que lui impose son mari et ceux, psychologiques, de sa bellemère. On a la représentation de deux types de femmes immigrées. D'une part, la belle-mère qui est la gardienne des traditions, comme dans Vivre au paradis, et, d'autre part, l'épouse qui représente la modernité, l'ouverture. Tout au long du film, ces deux femmes s'affrontent et s'efforcent d'imposer à l'autre leur avis. Le mari joue un rôle secondaire, il fera appliquer la loi de celle qui gagnera le combat.

Dans le film Vivre au paradis, c'est la tradition qui va gagner et qui va se maintenir, tandis que dans Inch'allah dimanche, c'est la modernité qui va s'imposer, car l'épouse triomphera et ouvrira la porte de la modernité à sa famille pour lui permettre de s'intégrer dans la vie française.

\section{Les modalités de rupture avec les origines}

Les problèmes que rencontrent les femmes immigrées dans le pays d'accueil ne sont pas exclusifs de la situation en France. Au Québec également des femmes tentent l'aventure de l'immigration. Dans ce pays francophone, au cours des dernières années, un grand nombre de films ont traité de l'immigration et plus particulièrement des problèmes posés par les communautés dérivées de l'immigration et de la politique multiculturaliste du pays. 
Un film nous paraît intéressant pour montrer différents aspects de l'immigration féminine au Québec. Il s'agit du film de Maryanne Zéhil, De ma fenêtre, sans maison (2006) où l'on nous montre la vie d'une femme libanaise installée au Québec. Le film expose la théorie selon laquelle l'unique possibilité d'adaptation dans un pays étranger consiste à rompre tous les ponts et à briser tous les liens avec le pays d'où on vient. Dans De ma fenêtre, sans maison, la protagoniste a quitté son pays pour pouvoir vivre une vie de "femme", libérée du rôle que lui impose la société libanaise dans laquelle elle vivait, et cela au prix de l'abandon de sa fille. Son désir de rompre avec toutes les traditions libanaises qui seraient susceptibles de l'empêcher d'être une femme libre l'a amenée à ne pas se mêler aux Libanais du Québec. En effet, les Libanais ont constitué une communauté dans laquelle subsiste le système hiérarchique hommefemme, caractéristique de la culture méditerranéenne.

Dans De ma fenêtre, sans maison De ma fenêtre, sans maison de Maryanne Zéhil, on trouve plusieurs types de femmes immigrées. Tout d'abord, la principale protagoniste qui a coupé les ponts avec tout ce qui peut la rapprocher de ses origines. Ensuite, une autre femme libanaise qui est mal dans sa peau parce qu'elle ne parvient ni à rompre avec les traditions de son pays, ni à s'adapter au pays qui l'a accueillie. Enfin, nous voyons la fille de la première qui arrive au Québec pour retrouver sa mère, et qui se sentira partagée entre la façon de vivre libanaise, et la vie québécoise pleine de liberté qui s'ouvre devant elle. Cette communauté libanaise n'est évidemment pas la seule au Québec où les immigrants, comme nous l'avons vu, se regroupent selon leurs origines et vivent en communautés repliées sur elles-mêmes, sans se mélanger ni entre elles ni avec les Québécois. Cette politique multiculturaliste pose actuellement beaucoup de problèmes à l'État et à la société canadienne ${ }^{(3)}$.

Parmi les films québécois qui prennent pour thème l'immigration féminine, nous nous attarderons sur le film de Guylaine Dionne, Serveuses demandées (2009). Le sujet porte sur le désespoir des femmes immigrées dont le visa n'a pas été renouvelé et sur le trafic de femmes qui s'ensuit. La protagoniste est une Brésilienne qui est venue au Québec pour suivre des études au terme desquelles l'État ne lui a pas renouvelé le visa. Voulant à tout prix rester au Québec, elle finit par travailler dans un club de danses exotiques où les filles, qui sont toutes des immigrées en situation illégale, 
acceptent de danser et de faire du strip-tease pour pouvoir rester en gagnant leur vie. Deux séquences sont particulièrement dramatiques et même déchirantes : dans l'une nous voyons passer, filmées en gros plan, chacune des filles qui racontent brièvement dans leur propre langue la trajectoire qui les a amenées à cette situation puis; dans l'autre, les mêmes filles racontent la même chose mais en français. Ce changement de langue est révélateur d'une intégration en voie de réalisation. Dans la première séquence, nouvellement arrivées, elles ne parlaient que leur langue maternelle. Dans la deuxième, après quelques années passées au Québec, elles démontrent une connaissance de la langue française qui témoigne d'une acculturation québécoise. Elles ne se sentent plus étrangères et demandent à pouvoir rester au Québec.

Ces jeunes femmes ou même ces jeunes filles, puisque leur âge va de 14 à 27 ans, sont arrivées pour faire des études ou pour travailler avec un contrat de travail comme femmes de ménage ou filles au pair. Mais leurs visas n'ont pas été renouvelés et elles se retrouvent, pour pouvoir rester et survivre, avec la seule chose dont elles disposent : leur corps. C'est le côté dramatique de la vie de ces femmes immigrées, qui finissent dans des clubs ou même sur le trottoir pour pouvoir survivre, que nous fait voir ce film. Ces femmes, qui sont arrivées en toute légalité, qui avaient un travail ou qui ont fait des études grâce à l'État canadien se retrouvent abandonnées par ce même État et deviennent des proies pour les mafias. On les voit faire une critique de la politique de régularisation des étrangers, du traitement réservé par le Québec aux sans-papiers.

\section{Communauratisme vs. assimilation : l'échec des politiques migratoires}

On a vu que la France et le Québec sont deux pays qui ont des politiques d'immigration différentes. Ces deux façons de traiter la même question sont bien représentées dans les films produits par ces deux pays. Dans les films français, les principaux problèmes qui se posent sont l'assimilation des familles et la dualité identitaire des enfants d'immigrés. L'image qui apparaît le plus souvent dans les films français est celle d'un immigré pauvre, qui est venu à la recherche de travail à une époque où la France avait besoin de main-d'ceuvre et qui a eu des problèmes d'intégration dans une société qui l'accueillait, mal le plus souvent, et où il a dû avoir affaire à des attitudes racistes, souvent dues au passé colonial français. Cette assimilation difficile de ces travailleurs qui ont été les premières victimes de la crise économique a eu une influence souvent décisive sur les enfants de la deuxième génération qui ont eu du mal à trouver leur identité et parfois à se sentir français. Dans les films québécois, les difficultés des enfants de la deuxième génération ne constituent pas un thème récurrent. Les 
problèmes qui se posent aux immigrés sont autres. Le multiculturalisme prôné par les autorités conduit les étrangers qui s'installent dans le pays à se réunir, selon leur origine, dans des communautés de quartier homogènes d'où ils ne sortent que très peu. Les problèmes se posent lorsque le travailleur immigré veut quitter ce cocon identitaire et rompre avec les coutumes de sa communauté et de sa famille pour s'intégrer dans l'espace public québécois. En effet, le repli sur soi induit par cette pratique communautariste ne favorise pas l'intégration, et les immigrés ont du mal à devenir des Québécois à part entière. Les metteurs en scène québécois montrent l'immigration d'une manière qui la relie peu à la pauvreté et au travail. Il s'agirait plutôt de montrer le déracinement, la tristesse, la pénurie morale dans laquelle se trouvent les immigrés du fait de la sévérité de la politique de régularisation des sans-papiers.

L'image de l'immigration que nous donnent tous ces films, aussi bien au Québec qu'en France, nous montre que les politiques migratoires n'ont pas complètement réussi. Bien que différents, les problèmes qui se posent aux immigrés dans les deux pays sont bien réels et le cinéma a su nous alerter et nous émouvoir en retraçant pour nous la vie difficile de ces personnes déracinées et qui ont tant de mal à se refonder dans un pays d'accueil.

\section{Filmographie}

- Bourlem Guerdjou, Vivre au paradis (1997).

- Christophe Ruggia Le Gone du Chaâba (1997).

- Dany Lafarrière, Comment conquérir l'Amérique (2004).

- Émile Gaudreault, Mambo Italiano (2004).

- Guylaine Dionne, Serveuses demandées (2009).

- Henri Verneuil, Mayrig (1991).

- Mahmoud Zemmouri, Beur, Blanc, Rouge (2005).

- Maryanne Zéhil, De ma fenêtre, sans maison (2006).

- Yamina Benguigui, Inch'Allah dimanche (2007).

\section{Bibliographie}

- Pilar González-Bernaldo, Manuela Martini, et Marie-Louise Pelus-Kaplan (dir.), Étrangers et sociétés : representations, coexistences, interactions dans la longue durée, Rennes, Presses Universitaires de Rennes, 2008.

- Hélène Greven-Borde, Jean Tournon, Les Identités en débat : intégration ou multiculturalisme ?, Paris, L'Harmattan, 2000.

- Michèle Lagny, Cine e Historia: problemas y métodos en la investigación cinematográfica, Barcelona, Bosch

Comunicación, 1997.

- Christophe Molaro, Violences urbaines et violences scolaires, Paris, L'Harmattan, 1998.

- Inés Molinaro, "Contexte et intégration. Les communautés allophones au Québec", in Globe. Revue internationale d'études québécoises, vol. 2, n², Montréal, université de Montréal, 1999, pp. 101-124.

\section{Notes}

1. Michèle Lagny, Cine e Historia: problemas y métodos en la investigación cinematográfica, Barcelona, Bosch Comunicación, 1997,pp. 181-181.

2. Gérard Noiriel, Le Creuset français : Histoire de l'immigration, $X I X^{e}-X X^{e}$ siècle, Paris, Seuil, 2006, p. 191.

3. Voir Hélène Greven-Borde, Jean Tournon, Les Identités en débat : intégration ou multiculturalisme?, Paris, L'Harmattan, 2000. 